\title{
Apparent negative values of Young's moduli of lattice materials under dynamic conditions
}

\begin{abstract}
Lattice materials are characterised by their equivalent elastic moduli for analysing mechanical properties of the microstructures. The values of the elastic moduli under static forcing condition are primarily dependent on the geometric properties of the constituent unit cell and the mechanical properties of the intrinsic material. Under a static forcing condition, the equivalent elastic moduli (such as Young's modulus) are always positive. When dynamic forcing is considered, the equivalent elastic moduli become functions of the applied frequency and they can be negative at certain frequency values. This paper, for the first time, explicitly demonstrates the occurrence of negative equivalent Young's modulus in lattice materials experimentally. Using additively manufactured titanium-alloy lattice metastructures, it is shown that the real part of experimentally measured in-plane Young's modulus becomes negative under a dynamic environment. In fact, we show that the onset of such negative Young's modulus in lattice materials can be precisely determined by capturing the subwavelength scale dynamics of the system. Experimental confirmation of the negative Young's moduli and the onset of the same as a function of frequency provide the necessary physical insights and confidence for its potential exploitation in various multi-functional structural systems and devices across different length scales.

Keywords: Negative Young's modulus; Lattice material; Dynamic analysis of honeycomb; Frequency-dependent elastic property; Onset of negative elastic moduli; Dynamics of additively manufactured lattice
\end{abstract}

\section{Introduction}

Lattice materials are a particular type of mechanical metamaterials, typically characterised by the periodicity of a unit cell. A crucial aspect of metamaterials is that the overall property is largely dependent on the geometric features of the periodic unit cells 
besides the intrinsic property of the constituent material. Intense research in recent years show truly exciting developments such as ultralight metamaterials [1] approaching theoretical strength limit [2], promise of exploiting stiffness most optimally and programmable shape modulation[3], pentamode materials [4] with cloaking mode [5], negative refraction elastic waves [6], elastic cloaking [7, 8], hyperbolic elastic metamaterials [9], negative Poisson's ratio (auxetic) materials [10], materials with negative effective elastic modulus [11] and negative mass density [12]. The interest in the current investigation is in the sub-acoustic range, which is typically considered to be below $\mathrm{kHz}$ range. In particular, this paper experimentally demonstrates the possibility of negative effective elastic moduli in 2D lattice metamaterials, and thereby investigates the onset of such negative Young's modulus as a function of frequency.

Lattice-like forms are available in abundance in naturally occurring as well as artificial materials and structures across different length-scales $[13,14,15,16,17,18]$. Methods to obtain effective properties (also know as homogenisation methods) of periodic elastic materials can be traced back to the classical work by Hashin and Shtrikman [19]. Exploiting periodic boundary conditions and mechanics of a unit cell, closed-form analytical expressions for equivalent elastic moduli for planar 2D cellular materials have been derived in $[15,20,21,22,23]$. Homogenisation of metamaterials with sub-wavelength dynamics needs to differ from the classical homogenisation approaches due to the fact that there are local resonators embedded in metamaterials. This has led to the development of dynamic homogenisation approaches $[24,25,26,27]$. The dynamic homogenisation can be viewed as a higher-order method [28] compared to the classical static homogenisation approaches. These homogenisation approaches are not strictly applicable when the unit cells are not periodic, as will be the case when random inhomogeneities are present in the metamaterial. To address this issue, the idea of 'representative unit cell element (RUCE)' was introduced in $[29,30]$ in the context of static homogenisation of cellular metamaterials. This approach is a step-change as it provides the geometric basis for considering inhomogeneities in cellular metamaterials and provides closed-form analytical expressions for equivalent (static) elastic properties. Homogenisation of continuum systems with random circular inclusions have been discussed recently $[31,32]$ for static problems.

Wave propagation in periodic structures [33] plays a crucial role in the analysis and design 


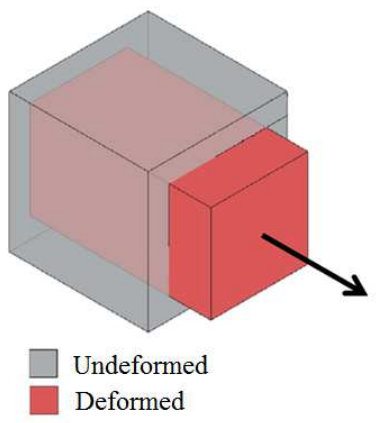

(a)
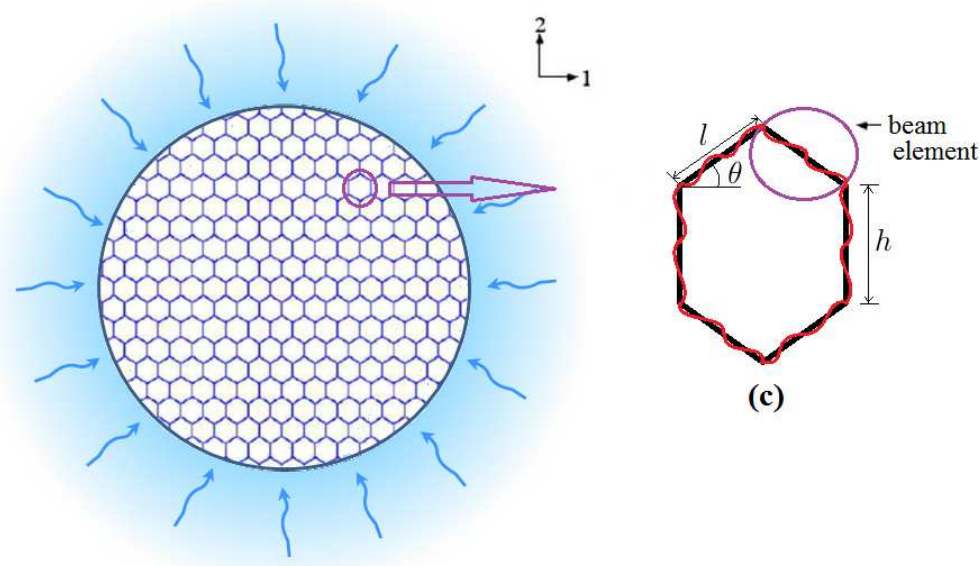

(c)

(b)

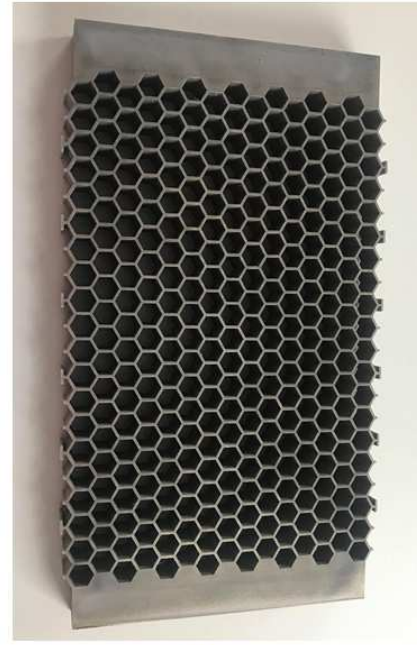

(d)

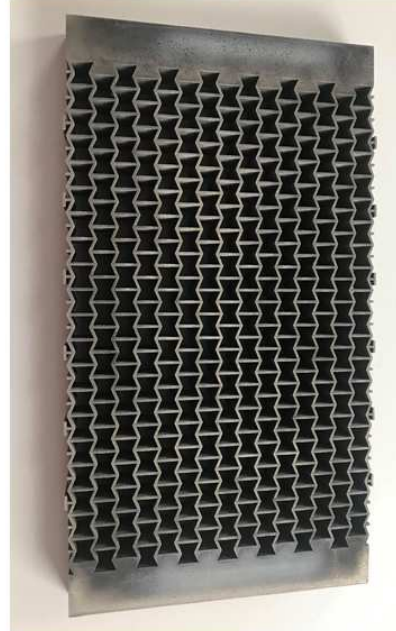

(e)

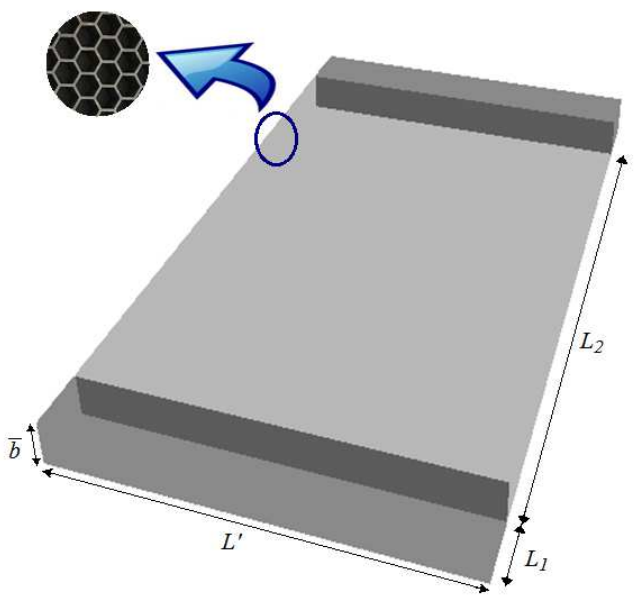

(f)

Figure 1: Lattice metamaterials under a vibrating environment. (a) Deformed shape of an equivalent continuum under uniaxial static (/quasi-static) deformation (b) Schematic representation of a hexagonal lattice microstructure under dynamic environment (for example, lattice microstructure as part of a larger host structure under wave propagation, vibration etc.). The curved arrows in this figure are symbolic representation of propagating wave. (c) Unit cell under a dynamic environment (d - e) Additively manufactured non-auxetic and auxetic samples of hexagonal lattice structures with intrinsic material as Titanium alloy Ti-6Al-4V (f) Equivalent continuum representation of the test specimen

of metamaterials. Extensive works have been undertaken since the mid 60's on dynamics of periodic structures [34] in aerospace engineering. The main motivation was to efficiently analyse large aerospace structures made of periodic units (e.g., periodically stiffened shell in an aircraft fuselage). Current computational methods for metamaterials [35] rely on the Floquet-Bloch theorem for wave propagation, which in turn is based on periodic boundary 
condition for a unit cell. Overall wave propagation behaviour depends on the dynamic characteristics of a unit cell and can be understood in terms of the band-gaps [36, 33]. Consequently, efficient analytical methods [37] and numerical methods for the computation of bandgaps of metamaterials have taken centre stage in most current research [38, 39, 40]. Classical wave propagation approaches were developed for undamped metamaterials. Few authors have considered damped metamaterials [41] where internal damping within a unit cell is considered explicitly [42, 43]. Bandgaps analysis for damped metamaterials needs the solution of a complex eigenvalue problem, which poses a computational challenge [44]. The dynamic behaviour of periodic structures can change drastically due to the presence of disorders [45]. Experimental works [46] show that in such cases a vibration can localise [47], similar to the phenomenon of Anderson's localisation [48] in atomic crystals.

Static elastic moduli of metamaterials differ from the elastic moduli under a vibrating condition [49]. Such dynamic elastic moduli are a function of the forcing frequency of vibration. The difference is due to the fact that deformation behaviour of the constituting members (such as the beam indicated in figure 1(c)) is different in static and dynamic environments. This essentially leads to the significant deviation in elastic moduli of lattice materials, which is actually a network of such constituent members. The enhanced elastic moduli has been proposed to be exploited in the optimum design of various structural systems (such as aircraft wings and turbine blades), which are subjected to vibration during the operational condition [49]. It can be shown that the elastic moduli could become negative at certain frequencies depending on the microstructural configuration. In a recent paper, the sub-wavelength scale dynamics in the deformation of a constituent beam element has been captured based on dynamic stiffness approach. This has led to the derivation of closed-form analytical limits of negative elastic moduli [50].

Negative Youngs moduli under a dynamic condition are directly relevant to developing tunable metamaterials for acoustic wave propagation [51]. This will lead to modulation of phase including complete inversion (/ switch) during wave propagation. Such an unusual behaviour in lattice materials potentially result in interesting applications such as acoustic cloak for waves, acoustic negative refraction, noise elimination and vibration reduction. The exploitation of this phenomenon can be utilized in a wide range of mechanical and acoustic systems where the modulation of dynamic properties are necessary. Another significant 
aspect arising from negative Youngs moduli is the low-frequency phase transformation and modulation. This makes the incredible possibility of practical applications at the sub-acoustic range such as at mechanical frequencies and even in seismic frequency ranges. Exploiting phase shifts and reversals, one can develop low-frequency adaptive programmable materials with applications in future aircraft, automotive, smart buildings and micro-mechanical devices and systems.

The possibility of effective negative elastic moduli in lattice materials has been explored mostly via theoretical approaches. Till date, little/ no experimental evidences have been put forward in the sub-acoustic range. This paper endeavours to outline the experimental methods and results aimed at understanding effective negative in-plane elastic moduli of 2D hexagonal lattices and the onset of such negative values. We have fabricated a hexagonal lattice made of a Titanium alloy with dimension $215 \times 115 \times 15 \mathrm{~mm}^{3}$ for this purpose. We consider a frequency range of $0-3000 \mathrm{~Hz}$ to obtain the effective in-plane elastic moduli of the lattice sample. Outline of the paper is as follows: theoretical background behind negative Young's moduli for cellular materials is discussed in section 2; section 3 covers the manufacturing of the honeycomb, the testing setup and main results arising from the experimental investigation; finally, a brief summary and concluding remarks on this work are presented in section 4 .

\section{Theoretical background for negative Young's moduli}

It is common practice to model lattice microstructures as continuous solid medium, wherein a unit cell based approach can be adopted to obtain the effective elastic moduli for the entire solid domain $[15,52,53,54,55]$. It has been shown theoretically that lattice materials with hexagonal honeycomb-like structures can exhibit negative Young's modulus under a dynamic environment [50]. The frequency corresponding to the onset of such negative Young's modulus in a honeycomb-like lattice material can be obtained based on dynamic stiffness approach in a multi-scale framework [50]

$$
\omega_{E_{1}, E_{2}}^{*} \approx 5.598 \frac{1}{l^{2}} \sqrt{\frac{E I}{m}}
$$

where $m, E$ and $I$ are the mass per unit length, intrinsic Young's modulus and area moment of inertia of the beam elements shown in figure 1(c) respectively, while the geometric dimension 
$l$ is indicated in the same figure. Here $\omega_{E_{1}, E_{2}}^{*}$ denotes the fundamental inflection frequency, where the Young's moduli (both $E_{1}$ and $E_{2}$ ) change sign from positive to negative. For a lightly damped microstructure, the equivalent Young's moduli will become negative for the first time beyond this frequency value. In other words, the above expression provides the lowest value of frequency to achieve negative Young's modulus. This will lead to a situation where the direction of steady-state dynamic response will be opposite to the forcing direction.

In the current paper we endeavour to undertake an experimental realization of the onset of negative Young's moduli. It can be noted that the inflection frequency is same for both longitudinal and transverse Young's moduli $\left(E_{1}\right.$ and $\left.E_{2}\right)$ and it only depends on the geometric parameter $l$ along with the intrinsic material properties. Thus this inflection frequency is invariant to the non-auxetic (refer to figure $1(\mathrm{~d})$ ) or auxetic (refer to figure $1(\mathrm{e})$ ) nature of the lattice geometry. The specimens of hexagonal honeycombs for carrying out experimental investigation are prepared using additive manufacturing with Titanium alloy Ti-6Al-4V as intrinsic material. It can be noticed in figure $1(\mathrm{~d}-\mathrm{e})$ that the specimens are manufactured with additional solid metallic tabs at both ends for experimental convenience. However, these metallic tabs would influence the dynamic properties of the hexagonal lattice structures leading to erroneous experimental results. In the analytical expression for inflection frequency (refer to Equation 1) the effect of such additional metallic tab has not been included. Thus to compare the analytical predictions with experimental results, it is necessary to include the influence of this metallic tab. Here we derive an analytical framework to take into account the error caused by the additional component attached to the honeycomb lattice.

An equivalent continuum representation of the specimen is shown in figure 1(f), where the middle portion of length $L_{2}$ has a lattice microstructure with equivalent Young's modulus $E^{\prime}$ and the two tabs of length $L_{1}$ each have Young's modulus $E^{\prime \prime}$. For the purpose of the current derivation of inflection frequency, we concentrate on the Young's modulus in direction-2 according to figure 1, which is essentially the in-plane direction perpendicular to the dimensions $L_{1}$ and $L_{2}$ in figure $1(\mathrm{f})$. Under a dynamic environment, the equivalent Young's modulus $E^{\prime}$ of a honeycomb lattice is given by [50]

$$
E^{\prime}(\omega)=\frac{D_{33}(h+l \sin \theta)}{l \bar{b} \cos ^{3} \theta}
$$


Here $\bar{b}$ is the thickness of honeycomb sheet as indicated in figure 1 (f). $D_{33}$ is a component of the dynamic stiffness matrix for a beam element [50]

$$
D_{33}=\frac{-E I b^{3}(c S+C s)}{(c C-1)}
$$

where $C=\cosh (b l), \quad c=\cos (b l), \quad S=\sinh (b l)$ and $s=\sin (b l) . \quad D_{33}$ is a frequency dependent complex quantity because $b$ is a function of $\omega$ and the damping factors [50]. The equivalent Young's modulus of the solid metallic tabs at the two ends of the lattice structure under dynamic environment is given by [56]

$$
E^{\prime \prime}=E \psi \cot \psi
$$

where $\psi^{2}=\frac{\omega^{2} \rho L^{\prime 2}}{E}$ and $\rho$ is the density of intrinsic material such that $m=\rho \times$ area of the connecting beam elements]. It can be noted here that the intrinsic Young's modulus of the lattice part in figure 1(f) and the Young's modulus of the solid metallic tabs are denoted by $E$ (both the parts are manufactured using same material).

If an in-plane force is applied along the two edges of the length $L\left(=2 L_{1}+L_{2}\right)$ in direction2 (i.e. perpendicular to the dimensions $L_{1}$ and $L_{2}$ ), the components of the forces shared by the honeycomb part of length $L_{2}$ and the metallic tabs of length $L_{1}$ each would be according to their respective axial stiffness.

$$
\frac{L \bar{b} E_{e q}}{L^{\prime}}=\frac{2 L_{1} \bar{b} E^{\prime \prime}}{L^{\prime}}+\frac{L_{2} \bar{b} E^{\prime}}{L^{\prime}}
$$

where $E_{e q}$ is the equivalent Young's modulus of the entire structure including the metallic tabs. From the above equation, the expression of $E_{e q}$ can be written as

$$
E_{e q}=E^{\prime} \frac{L_{2}}{L}+2 E^{\prime \prime} \frac{L_{1}}{L}
$$

Substituting $E^{\prime}$ and $E^{\prime \prime}$, the above expression can be modified as

$$
E_{e q}=D_{33} A+E \psi \cot \psi B
$$

where $A=\frac{(h+l \sin \theta) L_{2}}{\left(l \bar{b} \cos ^{3} \theta\right) L}$ and $B=\frac{2 L_{1}}{L}$. Expanding the expression of $D_{33}$ (by a Taylor series in the frequency parameter $\omega$; refer to Equation 3) and $\cot \psi$, the above equation becomes

$$
\begin{aligned}
E_{e q}(\omega) & =\left[12 \frac{E I}{l^{3}}-\frac{13}{35} m l \omega^{2}-\frac{59}{161700} \frac{l^{5} m^{2} \omega^{4}}{E I}+\cdots\right] A \\
& +\left[ \pm E \omega L^{\prime} \sqrt{\frac{\rho}{E}}\left(\frac{1}{ \pm \omega L^{\prime} \sqrt{\frac{\rho}{E}}}-\frac{1}{3}\left( \pm \omega L^{\prime} \sqrt{\frac{\rho}{E}}\right)-\frac{1}{45}\left( \pm \omega L^{\prime} \sqrt{\frac{\rho}{E}}\right)^{3}+\cdots\right)\right] B
\end{aligned}
$$


The value of $\omega$ for which $E_{e q}$ becomes zero can be obtained by solving $E_{e q}=0$, where retaining up to fourth order terms of $\omega$ (refer to Equation 8)

$$
\left(\frac{59}{161700} \frac{l^{5} m^{2}}{E I}\right) \omega^{4}+\left(\frac{13 m l}{35}+\frac{E L^{\prime 2} \rho}{3 E} \frac{B}{A}\right) \omega^{2}-\left(\frac{12 E I}{l^{3}}+E \frac{B}{A}\right)=0
$$

The solution of Equation 9 gives the frequency in the vicinity of $\omega=0$, where the Young's modulus changes sign from positive to negative.

As a special case of the above derivation, when no metallic tab is present at the two ends (i.e. $L_{1}=0$ and $L_{2}=L$ ), the parameter B becomes 0. Subsequently, Equation 9 reduces to

$$
\frac{59}{161700} \frac{l^{5} m^{2}}{E I} \omega^{4}+\frac{13 m l}{35} \omega^{2}-12 \frac{E I}{l^{3}}=0
$$

Solution of the above expression exactly matches with Equation 1. This observation essentially corroborates to further confidence in the current theoretical development. It is worthy to keep in mind at the current stage that Equation 9 can obtain fundamental inflection frequency including the effect of error induced due the presence of the two metallic tabs, which are designed essentially for operational convenience in the experimental setup. The current theoretical development of Equation 9 is solely to facilitate a comparison with the experimental outcome. The analytical framework presented in this section is valid for low cell wall thickness of the lattice structure. The in-plane dimensions of the experimental specimen are indicated in figure 2(b), while thickness in the out-of-plane direction is $0.015 \mathrm{~m}$. The intrinsic material properties of Titanium alloy Ti-6Al-4V are considered as: $E=110$ GPa and $\rho=4.7 \times 10^{3} \mathrm{~kg} / \mathrm{m}^{3}$. Using Equation 9, the fundamental inflection frequency is obtained as (discarding the negative and imaginary results for $\omega$ ) $\omega=1177 \mathrm{~Hz}$. In the following section, we will verify this value experimentally.

\section{Experimental investigation for the negative Young's moduli}

\subsection{Additive manufacturing of the honeycomb}

The Ti-6Al-4V lattice materials were additively manufactured on a Renishaw RenAM 500M, which is a Laser Powder Bed Fusion (L-PBF) process. The RenAM 500M system uses a 500W Ytterbium fibre laser to melt Ti-6Al-4V gas atomised powder onto a 250mm $\times$ $250 \mathrm{~mm}$ build plate, on a layer by layer basis, up to a maximum built height of $280 \mathrm{~mm}$. In this instance the Ti-6Al-4V powder was ELI grade and supplied by LPW. The parameters used in these builds were a power of $400 \mathrm{~W}$, a layer thickness of $60 \mu \mathrm{m}$, a point distance of 
$80 \mu \mathrm{m}$, an exposure time of $60 \mu \mathrm{s}$, and a hatch spacing of $100 \mu \mathrm{m}$. Both the non-auxetic lattice in figure $1(\mathrm{~d})$ and the auxetic lattice in figure 1(e) were built directly onto the base plate without any support structure, but with an additional $1 \mathrm{~mm}$ sacrificial layer. The lattices were then removed from the base plate using Electric Discharge Machining (EDM) so that the thickness $\bar{b}$ in figure 1 (f) was $15 \mathrm{~mm}$. The final dimension of the lattices in XY plane come to $215 \mathrm{~mm} \times 115 \mathrm{~mm}$. Residual stress is known to affect mechanical properties, however, no stress relieving post-build heat treatments were used in this instance, which resulted in a small spring-back deformation in the build direction after removal from the plate. Both machine parameters [57] and heat treatments [58] can be used in combination to reduce residual stress levels in the material and hence reduced distortion on removal from the base plate. Furthermore, it is also known that there will be a small amount of porosity $(<0.1 \%)$ which will also affect both elastic and plastic properties of the material, and this could be reduced using hot isostatic pressing [58].

\subsection{Testing setup}

The additively manufactured honeycomb structure is tested using an impact hammer. This is achieved with the aid of DataPhysics software and the 901 Series dynamic signal analyser to return the Frequency Response Functions (FRFs) between the force sensor in the hammer tip and the accelerometers. After some initial testings, a frequency bandwidth of $6400 \mathrm{~Hz}$ is used for the sample. Due to the higher natural frequencies, a stiff tip is used on the hammer in order to create a shorter pulse duration and increase the frequency range generated, and an exponential window was used to ameliorate the potential problem of leakage and improve the signal to noise ratio by reducing the influence of the noise long after the impact [59].

Boundary conditions for the experiment are a major consideration. Incorrect mounting of the structure for testing can dramatically change the results of the testing. In experimental analysis completely free boundary conditions can never be replicated; however, soft mounts were used to approximate free-free conditions. Hanging the structures so that the plane of the structure is vertical and suspended by elastic threads connected to each of the top corners ensures maximum freedom of movement for the structure during testing.

For the purpose of cross checking signals and to assist in verifying that the vibration 


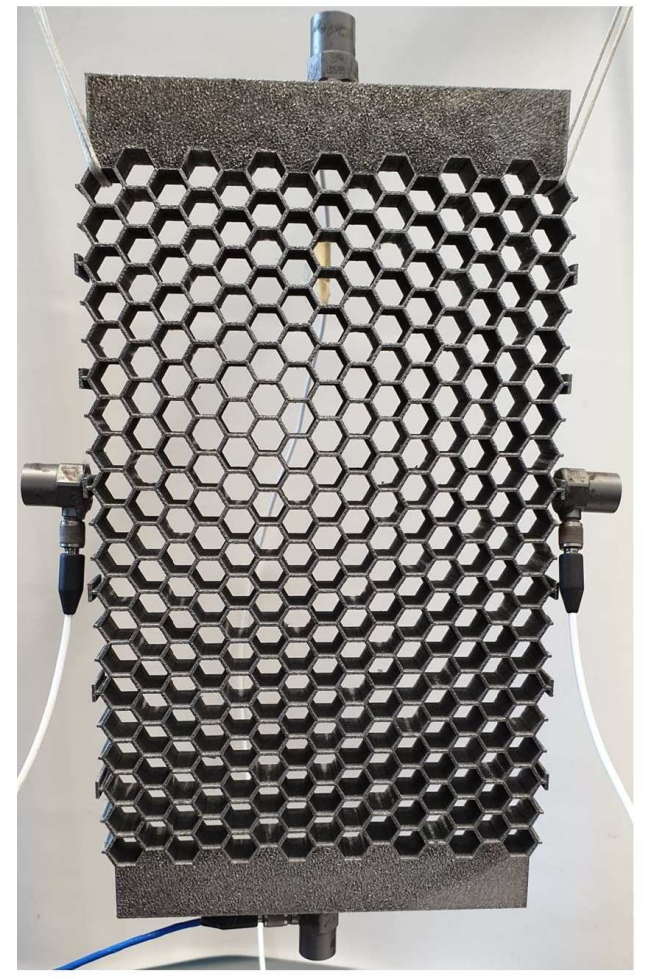

(a)

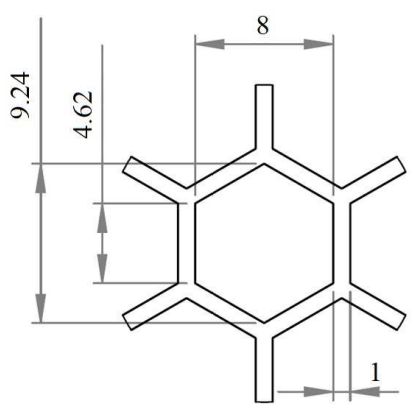

(b)

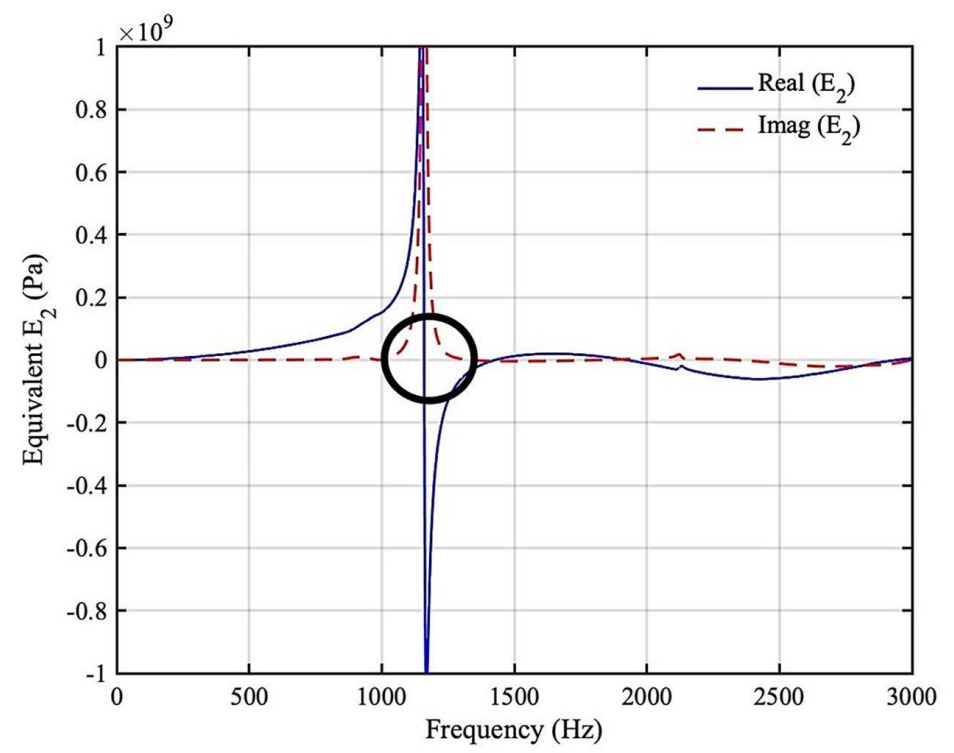

(c)

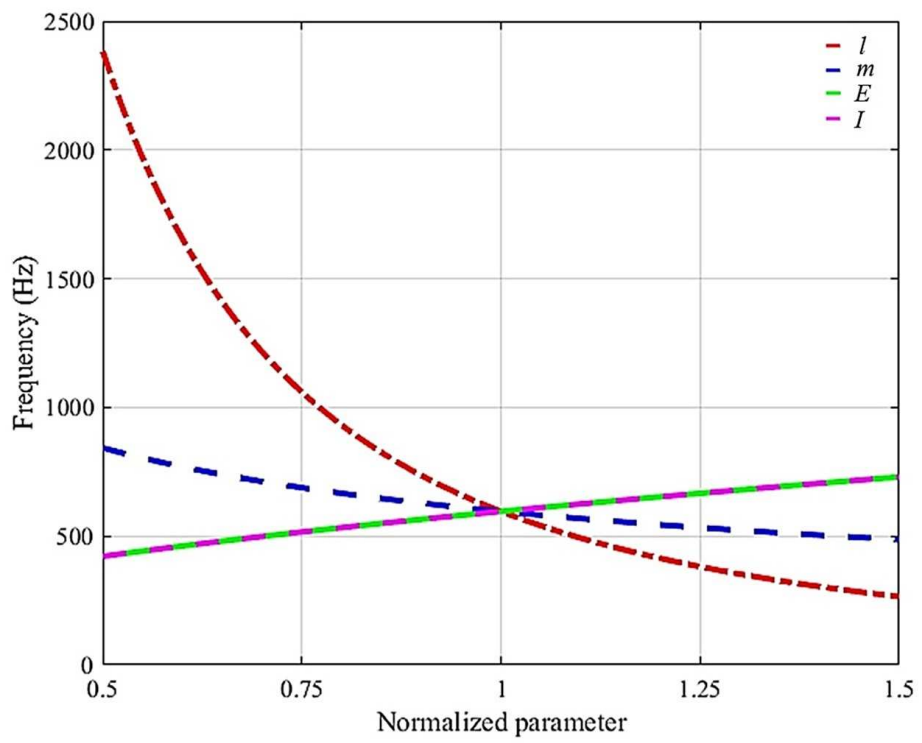

(d)

Figure 2: Onset of negative Young's moduli. (a) Description of the experimental setup (b) Dimensions (in c.m.) of a unit cell considered for the experimental investigation (c) Experimental results for variation of Young's modulus with frequency (real and imaginary components of $E_{2}$ are plotted as a function of frequency) (d) Dependence of the onset of negative Young's moduli on microstructural geometry and intrinsic material properties (Here the critical frequency for the onset of negative Young's moduli is plotted as a function of the geometric and material properties. These parameters are plotted along the abscissa in a normalized form with respect to the respective nominal values considered in the experimental investigations).

is fundamentally axial in nature, four accelerometers are attached to the structure. One accelerometer is placed at each edge as displayed in figure 2(a). These measure the acceleration of the structures in $\mathrm{g}$, when subject to impact from the hammer. Placement of these accelerometers on the structures are also considered. If they were placed too close to a node 
of a mode shape, that particular accelerometer could be a potential noise source, as it would experience low amplitudes. Here effect of the small additional mass caused by the physical attachment of sensors to the structure is neglected.

To obtain the in-plane axial response, the impact of the hammer is applied to the top of the honeycomb structure, as centrally as can be practically achieved. Using the accelerometers, accelerance (acceleration per unit force) frequency response functions (FRF) are produced. These are evaluated, determining the response to excitation vibration and thus the modal response at resonance. In total five channels of data have been stored. They include four accelerometers shown in figure 2(a) and the impact hammer. The real and imaginary parts of the frequency response for all the five channels have been stored for all the frequency points. The relative deformation of the lattice is obtained by subtracting the accelerometer reading of the opposite edges and dividing the resulting complex vector by frequency-square (note $A=-\omega^{2} X$, where $A$ is the acceleration and $X$ is the displacement). The effective dynamic strain is therefore obtained by dividing this quantity by the overall length of the lattice. The measured frequency dependent force is divided by the surface area of the top of the lattice to obtain the applied stress. The ratio of the effective stress and stain calculated this way gives the measured Young's modulus and is plotted in figure 2(c) by separating the real and imaginary parts.

\subsection{Results}

Experimental results for the real and imaginary parts of the frequency-dependent Young's modulus in direction 2 is plotted as a function of frequency in figure 2(c). We show in this figure experimentally that negative Young's modulus can be achieved in a dynamic environment. From the figure it can be noticed that real part of the Young's modulus changes sign from positive to negative at a frequency of $1161 \mathrm{~Hz}$, which is the onset of negative Young's modulus. It is interesting to note that the value is close to the theoretical prediction $(1177 \mathrm{~Hz})$ using Equation 9, as presented in the preceding section. This equation has been developed by incorporating the effect of two metallic tabs which are attached to the honeycomb panel for experimental convenience. In other words, since the prediction of Equation 1 is not directly comparable to the current experimental outcomes, the Equation 1 is modified further to incorporate the error induced by two additional metallic tabs. 
Close agreement between the modified theoretical framework and experimental outcomes essentially indicates the validity of Equation 1, which can predict the onset of negative Young's modulus without any influence of the metallic tabs. After obtaining enough confidence in the closed-form expression of fundamental inflection frequency (defined by the frequency where the elastic modulus changes sign for the first time [50]) based on the current experimental results, we investigate the effect of different material and geometric parameters that influence the onset of negative Young's modulus (both longitudinal and transverse). Figure $2(\mathrm{~d})$ shows the effect of two geometric parameters $(l$ and $I)$ and two intrinsic material properties $(m$ and $E$ ) on the fundamental inflection frequency. For the purpose of carrying out this numerical investigation, we have taken a range $[0.5 X 1.5 X]$ of the four parameters, where $X$ denotes the nominal value of the four parameters considered for carrying out the experiments. The abscissa of figure $2(\mathrm{~d})$ is normalized with respect to the respective $X$ values of each parameter. From the figure it is evident that the fundamental inflection frequency reduces with the increase in $l$ and $m$, while the sensitivity of $l$ is significantly higher compared to $m$. Both $E$ and $I$ can be noticed to have an opposite effect on the fundamental inflection frequency and their relative sensitivity is equal.

\section{Conclusions}

This paper presents an experimental investigation on the dynamic behaviour of lattice materials leading to the realization of the onset of negative Young's moduli. Under a static forcing condition, the equivalent elastic moduli of a material are always positive, which has been the traditional understanding of material behaviour for decades. However, it has recently been shown that the negative equivalent elastic moduli of lattice materials can be attained under a dynamic environment at certain frequency values. This paper attempts for the first time to demonstrate the occurrence of negative equivalent Young's modulus in lattice materials experimentally, and thereby identify the onset of such negative Young's modulus. Using hexagonal honeycomb lattices made of titanium alloy, it is shown that the real part of in-plane Young's modulus becomes negative under a dynamic environment. We have established that the onset of such negative Young's modulus in lattice materials can be precisely determined by capturing the sub-wavelength scale dynamics based on a modified analytical formulation including the effect of experimental setup. Thus the paper presents 
a comprehensive argument using both experimental investigation and a strong theoretical framework. The onset of negative Young's modulus is found to depend on certain material and geometric parameters of the lattice microstructure in a unique manner, which is numerically characterised as an integral part of this investigation. Although we have concentrated on hexagonal lattices in this paper, the experimental approach is generic in nature and could be extended to other lattices in two and three dimensions. In essence, the investigation presented in this article to identify the onset of negative Young's modulus in lattice materials would significantly contribute to the development of futuristic material microstructures at multiple length scales for exploiting the new dimensions of elastic moduli unravelled in the dynamic regime.

\section{Acknowledgements}

SA acknowledges the financial support from MSCA-IF-2017-799201. TM acknowledges the financial support form IIT Kanpur during this research work. NPL would like to thank the Welsh European Funding Office (WEFO) for the ASTUTE, ASTUTE 2020 and M2A funding which have enabled Swansea University to invest in an ALM machine. Further thanks go to the Welsh Assembly Government for the funds to set up the Materials Advanced Characterisation Centre (MACH1). The authors would also like to thank Dan Butcher for printing the builds.

\section{References}

[1] Zheng, X., Lee, H., Weisgraber, T. H., Shusteff, M., DeOtte, J., Duoss, E. B., Kuntz, J. D., Biener, M. M., Ge, Q., Jackson, J. A., Kucheyev, S. O., Fang, N. X., and Spadaccini, C. M. Ultralight, ultrastiff mechanical metamaterials. Science, 344(6190): 1373-1377, 2014.

[2] Berger, J. B., Wadley, H. N. G., and McMeeking, R. M. Mechanical metamaterials at the theoretical limit of isotropic elastic stiffness. Nature, 543(7646):533-537, 032017.

[3] Mukhopadhyay, T., Ma, J., Feng, H., Hou, D., Gattas, J. M., Chen, Y., and You, Z. Programmable stiffness and shape modulation in origami materials: Emergence of a distant actuation feature. Applied Materials Today, 19:100537, 2020. 
[4] Kadic, M., Buckmann, T., Stenger, N., Thiel, M., and Wegener, M. On the practicability of pentamode mechanical metamaterials. Applied Physics Letters, 100(19):191901, 2012.

[5] Buckmann, T., Thiel, M., Kadic, M., Schittny, R., and Wegener, M. An elastomechanical unfeelability cloak made of pentamode metamaterials. Nature Communications, 5:4130 EP -, 062014.

[6] Zhu, R., Liu, X. N., Hu, G. K., Sun, C. T., and Huang, G. L. Negative refraction of elastic waves at the deep-subwavelength scale in a single-phase metamaterial. Nature Communications, 5:5510 EP -, 112014.

[7] Milton, G. W., Briane, M., and Willis, J. R. On cloaking for elasticity and physical equations with a transformation invariant form. New Journal of Physics, 8(10):248, 2006.

[8] Stenger, N., Wilhelm, M., and Wegener, M. Experiments on elastic cloaking in thin plates. Physical Review Letters, 108:014301, Jan 2012.

[9] García-Chocano, V. M., Christensen, J., and Sánchez-Dehesa, J. Negative refraction and energy funneling by hyperbolic materials: an experimental demonstration in acoustics. Physical Review Letters, 112:144301, Apr 2014.

[10] Lakes, R. Foam structures with a negative poisson's ratio. Science, 235(4792):10381040, 1987.

[11] Fang, N., Xi, D., Xu, J., Ambati, M., Srituravanich, W., Sun, C., and Zhang, X. Ultrasonic metamaterials with negative modulus. Nature Materials, 5(6):452-456, 06 2006.

[12] Yang, Z., Mei, J., Yang, M., Chan, N. H., and Sheng, P. Membrane-type acoustic metamaterial with negative dynamic mass. Physical Review Letters, 101:204301, Nov 2008. doi: 10.1103/PhysRevLett.101.204301. URL https://link.aps.org/doi/10. 1103/PhysRevLett.101.204301.

[13] Mukhopadhyay, T. and Adhikari, S. Free vibration analysis of sandwich panels with randomly irregular honeycomb core. Journal of Engineering Mechanics, 10.1061/(ASCE)EM.1943-7889.0001153, 06016008, 2016.

[14] Mukhopadhyay, T. and Adhikari, S. Effective in-plane elastic properties of quasi-random spatially irregular hexagonal lattices. International Journal of Engineering Science, 119 (10):142-179, 2017. 
[15] Gibson, L. and Ashby, M. F. Cellular Solids Structure and Properties. Cambridge University Press, Cambridge, UK, 1999.

[16] Mahata, A. and Mukhopadhyay, T. Probing the chirality-dependent elastic properties and crack propagation behavior of single and bilayer stanene. Phys. Chem. Chem. Phys., 20:22768-22782, 2018.

[17] Chandra, Y., Mukhopadhyay, T., Adhikari, S., and Figiel, Ł. Size-dependent dynamic characteristics of graphene based multi-layer nano hetero-structures. Nanotechnology, 31(14):145705, 2020.

[18] Gupta, K. K., Mukhopadhyay, T., Roy, A., and Dey, S. Probing the compound effect of spatially varying intrinsic defects and doping on mechanical properties of hybrid graphene monolayers. Journal of Materials Science $\&$ Technology, In Press, 2020.

[19] Hashin, Z. and Shtrikman, S. A variational approach to the theory of the elastic behaviour of multiphase materials. Journal of the Mechanics and Physics of Solids, 11(2): 127 - 140, 1963. ISSN 0022-5096. doi: http://dx.doi.org/10.1016/0022-5096(63)90060-7. URL http://www.sciencedirect.com/science/article/pii/0022509663900607.

[20] El-Sayed, F. K. A., Jones, R., and Burgess, I. W. A theoretical approach to the deformation of honeycomb based composite materials. Composites, 10(4):209-214, 1979.

[21] Mukhopadhyay, T., Mahata, T., Zaeem, M. A., and Adhikari, S. Effective elastic properties of two dimensional multiplanar hexagonal nano-structures. 2D Materials, 4(2):025006:1-15, 2017.

[22] Mukhopadhyay, T., Mahata, A., Adhikari, S., and Zaeem, M. A. Effective mechanical properties of multilayer nano-heterostructures. Scientific reports, 7(1):15818, 2017.

[23] Mukhopadhyay, T., Mahata, A., Adhikari, S., and Asle Zaeem, M. Probing the shear modulus of two-dimensional multiplanar nanostructures and heterostructures. Nanoscale, 10:5280-5294, 2018.

[24] Willis, J. Exact effective relations for dynamics of a laminated body. Mechanics of Materials, 41(4):385 - 393, 2009. The Special Issue in Honor of Graeme W. Milton.

[25] Nemat-Nasser, S., Willis, J. R., Srivastava, A., and Amirkhizi, A. V. Homogenization of periodic elastic composites and locally resonant sonic materials. Physical Review B, 83: 104103, Mar 2011. doi: 10.1103/PhysRevB.83.104103. URL https://link.aps.org/ doi/10.1103/PhysRevB.83.104103. 
[26] Norris, A. N., Shuvalov, A. L., and Kutsenko, A. A. Analytical formulation of threedimensional dynamic homogenization for periodic elastic systems. Proceedings of the Royal Society of London A: Mathematical, Physical and Engineering Sciences, 468 (2142):1629-1651, 2012. doi: 10.1098/rspa.2011.0698.

[27] Craster, R. V., Kaplunov, J., and Pichugin, A. V. High-frequency homogenization for periodic media. Proceedings of the Royal Society of London A: Mathematical, Physical and Engineering Sciences, 2010. doi: 10.1098/rspa.2009.0612.

[28] Srivastava, A. Elastic metamaterials and dynamic homogenization: a review. International Journal of Smart and Nano Materials, 6(1):41-60, 2015.

[29] Mukhopadhyay, T. and Adhikari, S. Equivalent in-plane elastic properties of irregular honeycombs: An analytical approach. International Journal of Solids and Structures, 91(8):169-184, 2016.

[30] Mukhopadhyay, T. and Adhikari, S. Stochastic mechanics of metamaterials. Composite Structures, 162:85-97, 2017.

[31] Pivovarov, D. and Steinmann, P. Modified sfem for computational homogenization of heterogeneous materials with microstructural geometric uncertainties. Computational Mechanics, 57(1):123-147, Jan 2016.

[32] Pivovarov, D. and Steinmann, P. On stochastic fem based computational homogenization of magneto-active heterogeneous materials with random microstructure. Comput. Mech., 58(6):981-1002, December 2016. ISSN 0178-7675.

[33] Brillouin, L. Wave Propagation in Periodic Structures. Dover Publications, New York, USA, 1953.

[34] Mead, D. Wave propagation in continuous periodic structures: research contributions from southampton, 1964-1995. Journal of Sound and Vibration, 190(3):495 - 524, 1996.

[35] Hussein, M. I., Leamy, M. J., and Ruzzene, M. Dynamics of phononic materials and structures: historical origins, recent progress, and future outlook. Applied Mechanics Reviews, 66(4):040802-38, 052014.

[36] Deymier, P. A. Acoustic Metamaterials and Phononic Crystals. Springer Series in Solid-State Sciences, Vol. 173. Springer, New York, USA, 2013.

[37] Bigoni, D., Guenneau, S., Movchan, A. B., and Brun, M. Elastic metamaterials with inertial locally resonant structures: Application to lensing and localization. Physical 
Review B, 87:174303, May 2013.

[38] Hussein, M. I. Reduced bloch mode expansion for periodic media band structure calculations. Proceedings of the Royal Society of London A: Mathematical, Physical and Engineering Sciences, 465(2109):2825-2848, 2009.

[39] Sugino, C., Leadenham, S., Ruzzene, M., and Erturk, A. On the mechanism of bandgap formation in locally resonant finite elastic metamaterials. Journal of Applied Physics, 120(13):134501, 2016.

[40] Palermo, A. and Marzani, A. Extended bloch mode synthesis: Ultrafast method for the computation of complex band structures in phononic media. International Journal of Solids and Structures, 100:29-40, 2016.

[41] Hussein, M. I. Theory of damped bloch waves in elastic media. Physical Review B, 80: 212301, Dec 2009.

[42] Hussein, M. I. and Frazier, M. J. Metadamping: An emergent phenomenon in dissipative metamaterials. Journal of Sound and Vibration, 332(20):4767 - 4774, 2013. ISSN 0022-460X. doi: http://dx.doi.org/10.1016/j.jsv.2013.04.041. URL http://www . sciencedirect.com/science/article/pii/S0022460X13003805.

[43] Yu, F., Collet, M., Ichchou, M., Lin, L., Bareille, O., and Dimitrijevic, Z. Enhanced wave and finite element method for wave propagation and forced response prediction in periodic piezoelectric structures. Chinese Journal of Aeronautics, 30(1):75-87, FEB 2017.

[44] Phani, A. S. and Hussein, M. I. Analysis of damped bloch waves by the rayleigh perturbation method. Journal of Vibration and Acoustics, 135(4):041014-11, 2013.

[45] Hodges, C. Confinement of vibration by structural irregularity. Journal of Sound and Vibration, 82(3):411 - 424, 1982.

[46] Hodges, C. H. and Woodhouse, J. Vibration isolation from irregularity in a nearly periodic structure: Theory and measurements. The Journal of the Acoustical Society of America, 74(3):894-905, 1983.

[47] Pierre, C. and Cha, P. D. Strong mode localization in nearly periodic disordered structures. AIAA Journal, 27(2):227-241, 1989.

[48] Anderson, P. W. Absence of diffusion in certain random lattices. Physical Review, 109: 1492-1505, Mar 1958. 
[49] Mukhopadhyay, T., Adhikari, S., and Alu, A. Probing the frequency-dependent elastic moduli of lattice materials. Acta Materialia, 165:654 - 665, 2019.

[50] Mukhopadhyay, T., Adhikari, S., and Alu, A. Theoretical limits for negative elastic moduli in subacoustic lattice materials. Phys. Rev. B, 99:094108, 2019.

[51] Hou, Z. and Assouar, B. M. Tunable solid acoustic metamaterial with negative elastic modulus. Applied Physics Letters, 106(25):251901, 2015.

[52] Mukhopadhyay, T. and Adhikari, S. Effective in-plane elastic properties of auxetic honeycombs with spatial irregularity. Mechanics of Materials, 95:204 - 222, 2016.

[53] Mukhopadhyay, T., Adhikari, S., and Batou, A. Frequency domain homogenization for the viscoelastic properties of spatially correlated quasi-periodic lattices. International Journal of Mechanical Sciences, 150:784-806, 2019.

[54] Zhang, H., Wang, Y., and Kang, Z. Topology optimization for concurrent design of layer-wise graded lattice materials and structures. International Journal of Engineering Science, 138:26-49, 2019.

[55] Khakalo, S., Balobanov, V., and Niiranen, J. Modelling size-dependent bending, buckling and vibrations of $2 \mathrm{~d}$ triangular lattices by strain gradient elasticity models: applications to sandwich beams and auxetics. International Journal of Engineering Science, $127: 33-52,2018$.

[56] Leung, A. Dynamic Stiffness and Substructures. Springer-Verlag, London, UK, 1993.

[57] Sillars, S. A., Sutcliffe, C. J., Philo, A. M., Brown, S. G. R., Sienz, J., and Lavery, N. P. The three-prong method: a novel assessment of residual stress in laser powder bed fusion. Virtual and Physical Prototyping, 13(1):20-25, 2018.

[58] Lavery, N., Cherry, J., Mehmood, S., Davies, H., Girling, B., Sackett, E., Brown, S., and Sienz, J. Effects of hot isostatic pressing on the elastic modulus and tensile properties of 3161 parts made by powder bed laser fusion. Materials Science and Engineering: A, 693:186 - 213, 2017.

[59] Ewins, D. Modal testing: theory, practice, and application. Mechanical engineering research studies: Engineering dynamics series. Research Studies Press, Baldock, 2000. 